\title{
An Overview of the Tectonic Evolution of the Indochina block and Granitoid Emplacement, particularly in the central and south Vietnam
}

\author{
Nguyen Anh Tuan ${ }^{1,2,}{ }^{*}$, Ngo Tran Thien Quy ${ }^{1}$, Vu Thi Hao ${ }^{1}$, Pham Minh ${ }^{1}$
}

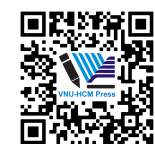

Use your smartphone to scan this QR code and download this article

${ }^{1}$ University of Science, Vietnam National University Ho Chi Minh City

${ }^{2}$ University of Technology, Vietnam National University Ho Chi Minh City

Correspondence

Nguyen Anh Tuan, University of Science, Vietnam National University Ho Chi Minh City

University of Technology, Vietnam National University Ho Chi Minh City

Email:nanhtuan@hcmut.edu.vn

History

- Received: 2020-04-07

- Accepted: 2020-07-14

- Published: 2020-08-24

DOI : 10.32508/stdj.v23i3.2062

\section{Check for updates}

\section{Copyright}

(c) VNU-HCM Press. This is an openaccess article distributed under the terms of the Creative Commons Attribution 4.0 International license.

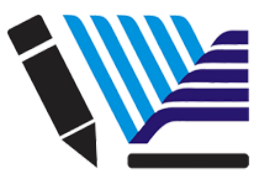

VNU-HCM Press

\begin{abstract}
Introduction: Vietnam is mainly located within the Indochina block in Southeast Asia. Asmall northern part of Vietnam belongs to the South China block, the southwest part liesadjacent to the Sibumasu block and opens to the East Sea on the east side. Tectonicactivities in Vietnam were very complicated they relate to intense interactions betweenmany geological blocks at different times. Magmatic emplacement is the final and instantproduct of tectonic activities. Methods: Geochemical data analysis from rock samples withinVietnam collected by other researchers has been reused in the scope of this study to verifythe relation between tectonic evolutions and their granitic magmatism. GCD (GeochemicalData Toolkit), an R language program for handling and recalculation of geochemical data. Results: Geochronology and geotectonic model derived from rock analysis have beenascertained main tectonic evolutions of the Indochina. The current granitoidclassification in Vietnam mostly based on petrographical studies. The Nui Cam granitoid isbeing classified as Deo Ca, Dinh Quan granitoid. However, based on trace elements, they aredifferent. They may belong to different granitoid system. Conclusion: Major tectonic eventswithin the Indochina block are well supported by the nature of granitoid emplacements. Petrological studies of these magmatic rocks would bring out valuable information toconfirm and clearly understand the tectonic evolutions of the region. Igneous rocksclassification must based on tectonic fundamental instead of petrographical studies.

Key words: Vietnam, Indochina, tectonic, granitoid emplacement
\end{abstract}

\section{INTRODUCTION}

The tectonic activities of the Indochina block and surrounded geological blocks are much more complex. The geological boundaries, defined as the tectonic sutures, where two geological blocks welded together, have been reported in many different places throughout the Indochina block. At least three main sutures have been found, and they are:

1. The Ordo-Silurian Tam Ky - Phuoc Son resulted from the assimilation of South China and Indochina blocks;

2. The Middle Triassic Sông Mã suture resulted from the welding of the Indochina and South China blocks; and

3. The Late Triassic suture resulted from the amalgamation of the Indochina and Sibumasu blocks.

Some of these sutures have been intensively studied, such as Sông Mã suture, the other just begins with primarily study while the Sibumasu and Indochina block while much of studies have been reported in
Thai Land, Cambodia, Malaysia, Laos, but it's almost ignored in Viet Nam. In this paper, with the other researchers' authorization, granitoid rocks petrochemical data collected in the central and south Vietnam were used and reprocessed in tectonic purposes to support the presence of these tectonic features in the Indochina block.

\section{A review of the tectono- magmatism}

An oceanic plate sinks beneath a continental plate; it gradually moves deeper to the hot dense mantle core. Under the increased heat and pressure conditions, it begins to melt itself and produce a magma fluid. But the most significant mechanism in the process is the release of water into the mantle by metamorphosing of hydrated serpentinites of the oceanic crust. The amount of $\mathrm{H} 2 \mathrm{O}$ then reduces the melting point of the surrounding mantle. A partial melt of the mantle occurs and generates magma fluid ${ }^{1,2}$. That magma fluid produced within the mantle is lighter compare to the surrounding environment moves up into the continental crust where it resides in the magma chambers. 
Inside the magma chamber hot magma cooler and solidifies and forms granitoid pluton within the crust, also some amount of $\mathrm{SiO} 2$ rich magma differentiated within the magma chamber could escape to the surface and forms an acidic volcanic chain.

Granitoid rocks have been well studied for a long time; their classification is varied and depends on the scope of researchs. In general, a classification of granitoid rocks based on tectonic setting can be seen as the studies of Pitcher (1983, 1993), Barbarin (1990) Figure 2.

In a basic and simple model of convergent tectonic between India and Eurasia plates, different types of granitoid forming through each stage of the evolution Figure 3.

(A) Initial subduction of India oceanic plate beneath Eurasia plate; VAG I type granitoid produced from the partial melt of mafic material of the oceanic crust itself and from the surrounding mantle; also $S$ type granitoid forming as mixing of mantle magma with continental crust material;

(B) Further subduction of India into the core of Eurasia, India continental crust closer to India, magma produced from mantle cease stop;

(C) The final stage of collision, heat caused by decaying radioactive materials within the crust melt felsic continental materials and produce a COLG and WPG type granitoid.

\section{Tectonic evolution of the Indochina block} from Devo-Silurian to Cretaceous

Separated from the northeastern part of the Gondwana supercontinent in the southern hemisphere began Devonian time, through different phases of the opening of the Tethyan sea, small continental blocks gradually moved northward to the Eurasia continent and finally assembled together to the south and southeast Asia by the Cretaceous time during long and complex geological activities. The Indochina block (e.g., Vietnam) has at least three main tectonic events that have been recognized in relation to complicated tectonic evolutions with other blocks in the region.

\section{Caledonian orogeny}

Beginning of Devonian with the opening of the Paleo-Tethyan sea, numerous small geological blocks such as Indochina, South China, North China, Tarim,...drifted away from Gondwanaland supercontinent and move northward. Silurian fish fossils have been distributed within the South China block, also discovered in Vietnam (Indochina block) ${ }^{3}$. It is suggested that these two blocks stay close to each other during Silurian time. No connected marine fossils have been reported since early Devonian time, it is suggested toward Devonian time, these two blocks connected and welded together. The amalgamation could happen toward the end of Devonian or Early Permian to form the Cathaysialand ${ }^{4}$ Figure 4 . However, a small sea branch believed to be presented between these blocks.

This Devonian tectonic event is referred to Caledonian Orogeny ${ }^{5}$, although this name is inappropriate to be applied to Asia, it has affected different parts of China and particularly in South China. In Vietnam, tectonic events reported by the Early Paleozoic are not well recognized. However, Devonian granitoids have been reported in the central area of Vietnam (Kontum Massif, Dai Loc Massif, Song Chay Massif ...) (Figure 5). It is highly possible that the tectonic event is largely distributed throughout Vietnam and likely is suitable to use as Caledonian orogeny.

\section{Indosinian orogeny and the amalgamation of Sibumasu/Indochina}

The Indochina block finally welded to the South China block by the middle Triassic and was named as Indosinian orogeny. Nature of the evolution between these two blocks is still in debate, it may be purely subduction of South China block under Indochina block from north to south ${ }^{6}$ Figure 6 , or it may be in the opposite direction by Indochina block from south to north under South China block ${ }^{7}$ Figure 7.

Another tectonic model also being suggested as the reactivation of an old suture ${ }^{8}$ Figure 8 . The Sibumasu block which separated from Gonwanaland by the opening of the Meso-Tethyan ocean at late Permian moved northward and docked to the southeast side of the Indochina block. This tectonic activity has reactivated the old suture which situated here between the two blocks and completely amalgamated them by middle Triassic time

\section{Sibumasu/Indochina Orogeny}

Moved northward after separated from Gondwanaland by early Permian, the Sibumasu came and docked in the southwest side of Indochina, also Tarim and North China, South China ... blocks. Different stages of tectonic evolution were reported in a long time span from Early Permian to Early Jurassic, specified in Sibumasu and Indochina blocks ${ }^{9}$ Figure 9 , associated with different granitoid emplacements in Thailand, Malaysian peninsula, Cambodia and southwest Vietnam Figure 10 . 


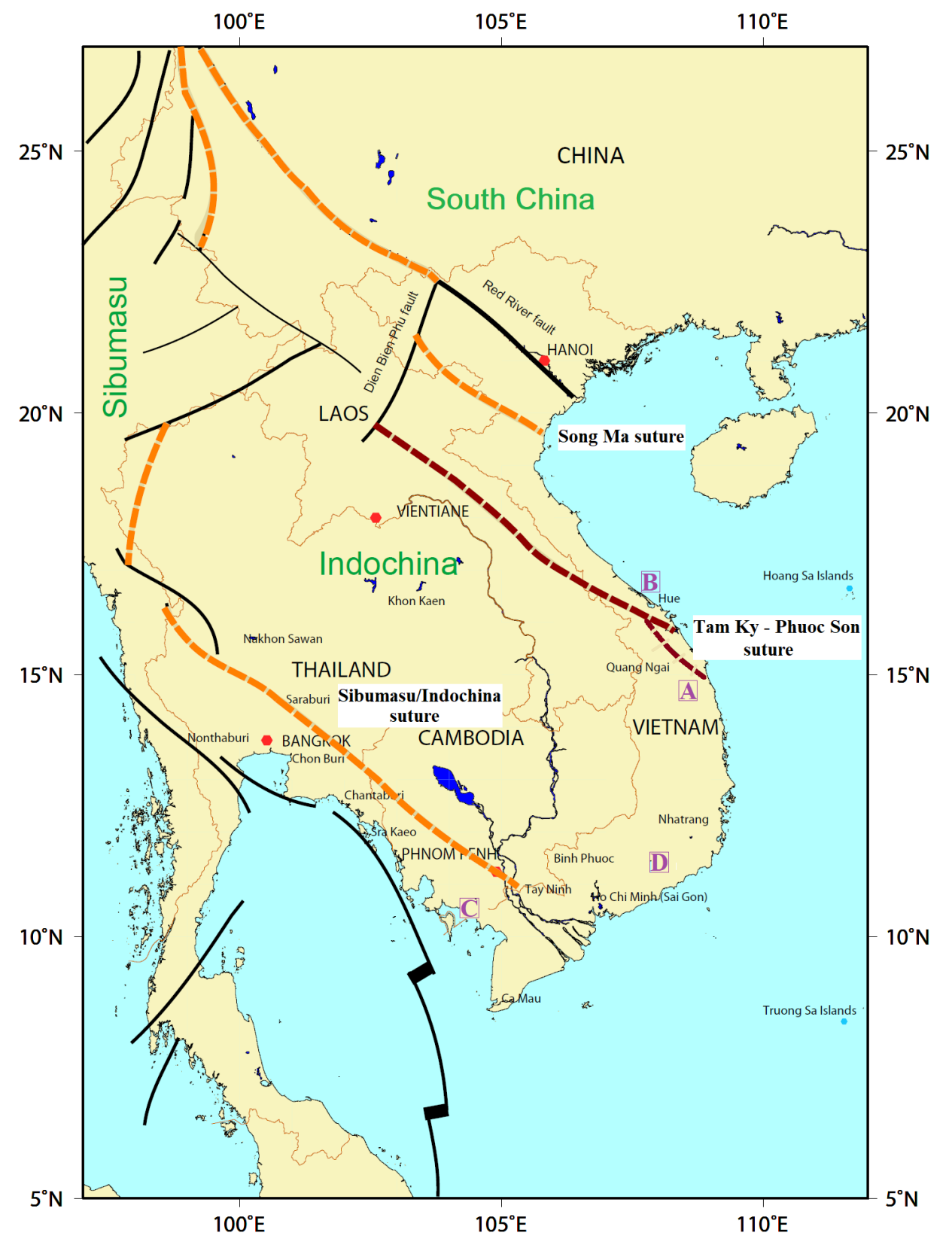

Figure 1: Location of Indochina/South China/Sibumasu blocks and main tectonic features (modified from Faure, 2008). 
A classification of granitoid rocks based on tectonic setting

\begin{tabular}{|c|c|c|c|c|c|c|}
\hline \multirow[b]{2}{*}{$\begin{array}{c}=\text { granitoid } \\
\text { magma }\end{array}$} & \multicolumn{3}{|c|}{ OROGENIC } & \multirow[b]{2}{*}{\begin{tabular}{|l|} 
TRANSITIONAL \\
$\begin{array}{c}\text { Post-Orogenic } \\
\text { Uplift/Collapse }\end{array}$ \\
$\begin{array}{c}\text { decompression } \\
\text { motting }\end{array}$
\end{tabular}} & \multicolumn{2}{|c|}{ ANOROGENIC } \\
\hline & $\begin{array}{l}\text { Ocea } \\
\text { Island }\end{array}$ & Arc & $\begin{array}{l}\text { Continental } \\
\text { Collision }\end{array}$ & & $\begin{array}{l}\text { Contintntal } \\
\text { Rifting. Hot Spot }\end{array}$ & $\begin{array}{c}\text { Mid-Ocean Ridge, } \\
\text { Ocean Islands } \\
\text { hot spot } \\
\text { plume }\end{array}$ \\
\hline Examples & $\begin{array}{c}\text { Bougainville, } \\
\text { Solomon Islands, } \\
\text { Papua New Guinea }\end{array}$ & $\begin{array}{c}\text { Mesozoic Cordilleran } \\
\text { batholiths of wost } \\
\text { Americas } \\
\text { Gander Terrane }\end{array}$ & $\begin{array}{l}\text { Manasiu and Lhotse } \\
\text { of Nepal, Amorican } \\
\text { Massif of Brittany }\end{array}$ & \begin{tabular}{|c|} 
Late Caledonian \\
Plutons of Britain, \\
Basin and Range, \\
late Variscan, early \\
Northern Proterozoic \\
\end{tabular} & \begin{tabular}{|c|} 
Nigerian ring \\
complexes, Oslo \\
rift, Britiss Tertiary \\
lgneous Province, \\
Yellowstone hotspot
\end{tabular} & $\begin{array}{l}\text { Oman and Troodos } \\
\text { ophiolites; } \\
\text { Icoland, Asconsion, } \\
\text { and Reunion Island } \\
\text { intrusives }\end{array}$ \\
\hline $\begin{array}{c}\text { Geo- } \\
\text { chemistry }\end{array}$ & $\begin{array}{c}\text { Calc-alkaline > thol. } \\
\text { M-type \& I-M hybrid } \\
\text { Motaluminous }\end{array}$ & $\begin{array}{c}\text { Calc-alkaline } \\
\text { I-type > S-type } \\
\text { Met-Al to sit. Per-Al }\end{array}$ & $\begin{array}{l}\text { Calc-alkaline } \\
\text { S-type } \\
\text { Poraluminous }\end{array}$ & $\begin{array}{c}\text { Calc-akaline } \\
\text { 1-type S-type (A-type) } \\
\text { Motalum. to Peralum }\end{array}$ & $\begin{array}{c}\text { Alkaline } \\
\text { A-type } \\
\text { Peralkaline }\end{array}$ & $\begin{array}{c}\text { Tholeilitic } \\
\text { M-type } \\
\text { Motaluminous }\end{array}$ \\
\hline $\begin{array}{l}\text { Rock } \\
\text { types }\end{array}$ & $\begin{array}{l}\text { qtz-diorite in } \\
\text { mature arcs }\end{array}$ & $\begin{array}{l}\text { tonalite \& granodior. } \\
>\text { granite or gabbro }\end{array}$ & $\begin{array}{l}\text { migmatites \& } \\
\text { levoogranite }\end{array}$ & $\begin{array}{c}\text { bimodal granodiorite } \\
\text { + diroite-gabbro }\end{array}$ & $\begin{array}{l}\text { Granite, syenite } \\
\text { + diorite-gabbro. }\end{array}$ & Plagiogranite \\
\hline \begin{tabular}{|c|} 
Associated \\
Minerals
\end{tabular} & $\mathrm{Hbl}>\mathrm{Bt}$ & $\mathrm{Hbl,} \mathrm{Bt}$ & $\begin{array}{l}\text { Bt, Ms, Hbl, Grt, } \\
\text { Als, Crd }\end{array}$ & $\mathrm{Hbl}>\mathrm{Bt}$ & \begin{tabular}{|c|}
$\mathrm{Hbl}$, Bt, aegirine \\
tayalite, Rbk, artved.
\end{tabular} & $\mathrm{Hbl}$ \\
\hline \begin{tabular}{|l|}
$\begin{array}{l}\text { Associated } \\
\text { Volcanism }\end{array}$ \\
\end{tabular} & $\begin{array}{c}\text { Island-arc basalt } \\
\text { to andesite }\end{array}$ & $\begin{array}{c}\text { Andesite and dacite } \\
\text { in oreat volume }\end{array}$ & often lacking & basalt and rhyolite & $\begin{array}{l}\text { alkali lavas, tuffs, } \\
\text { and caldera infill } \\
\end{array}$ & $\begin{array}{c}\text { MORB and ocean } \\
\text { island hasat? }\end{array}$ \\
\hline $\begin{array}{c}\text { Clossification } \\
\text { Barbarin } \\
(1990)\end{array}$ & $\begin{array}{c}T_{u} \\
\text { tholeitte island arc }\end{array}$ & $\begin{array}{c}\mathrm{H}_{a} \\
\text { hybrid calc-akaline }\end{array}$ & $\underset{\text { continental types }}{\mathrm{C}_{s T} \mathrm{C}_{\mathrm{ar}} \mathrm{C}_{a}}$ & \begin{tabular}{|c|}
$\mathrm{H}_{L}$ \\
hybrid late orogenic \\
\end{tabular} & $\begin{array}{c}\text { A } \\
\text { alkaline }\end{array}$ & \begin{tabular}{|c|}
$\mathrm{T}_{\mathrm{OR}}$ \\
tholeilte Ocean ridge
\end{tabular} \\
\hline $\begin{array}{c}\text { Pearce } \\
\text { ot al. (1984) }\end{array}$ & \multicolumn{2}{|c|}{ VAG (volcanic arc granites) } & \multicolumn{2}{|c|}{ COLG (collision granites) } & \multicolumn{2}{|c|}{$\begin{array}{c}\text { WPG and ORG } \\
\text { (within plate and ocean ridge granites) }\end{array}$} \\
\hline $\begin{array}{c}\text { Maniar \& } \\
\text { Piccoli (1989) } \\
\end{array}$ & \begin{tabular}{|c|} 
IAG \\
island arc granite \\
\end{tabular} & $\begin{array}{c}\text { CAG } \\
\text { contin. arc granite }\end{array}$ & $\begin{array}{c}\text { CCG } \\
\text { cont. collision gran. } \\
\end{array}$ & $\begin{array}{c}\text { POG } \\
\text { post-orogenic gran. }\end{array}$ & \begin{tabular}{|c|} 
RRG CEUG \\
ritt \& aborted/hotspot
\end{tabular} & \begin{tabular}{|c|} 
OP \\
ocean plagiogranite \\
\end{tabular} \\
\hline Origin & $\begin{array}{l}\text { Partar meiting or } \\
\text { mantle-derived } \\
\text { mafic underplate }\end{array}$ & $\begin{array}{l}\text { ma or mantie-oeriveg } \\
\text { mafic underplate + } \\
\text { crustal contribution }\end{array}$ & $\begin{array}{l}\text { Partal melting } \\
\text { of recycled } \\
\text { crustal material }\end{array}$ & $\begin{array}{c}\text { Panial melting of } \\
\text { lower crust+ mantle } \\
\text { and mid-crust contrib }\end{array}$ & $\begin{array}{l}\text { Pantial moting of } \\
\text { mantle and/or lower } \\
\text { crust (anhydrous) }\end{array}$ & $\begin{array}{c}\text { Partial melting of } \\
\text { mantle and frac- } \\
\text { tonal crystallization }\end{array}$ \\
\hline \begin{tabular}{|c} 
Melting \\
Mechanism
\end{tabular} & \multicolumn{2}{|c|}{\begin{tabular}{|l|} 
Subduction energy: transfer of fluids and \\
dissolved species from slab to wodge. \\
Melting of wedge, transter of heat upward
\end{tabular}} & $\begin{array}{l}\text { Tectonic thickening } \\
\text { plus radiogenic } \\
\text { crustal hea }\end{array}$ & $\begin{array}{l}\text { Crustal heat plus } \\
\text { mantle heat (rising } \\
\text { asthen. + magmas) }\end{array}$ & \multicolumn{2}{|c|}{ Hot spot and/or adiabatic mantle rise } \\
\hline
\end{tabular}

After Pitcher $(1983,1993)$, Barbarin (1990)

Figure 2: Tectonic evolution and granitoid emplacements after Pitcher $(1983,1993)$, Barbarin (1990).

\section{Yanshangnian orogeny}

Cretaceous time, a major tectonic event occurs on the east side, the Paleo_Pacific oceanic crust subducted beneath the Eurasia continent ${ }^{10}$. Numerous granitoid pluton largely distributed along the eastern coast of Asia from the north in China as Yanshannian granitoid toward the south in Vietnam as Truong Son granitoid Figure 11 .

Recent studies in the southern part, in Vietnam, there is not only a simple subduction of Paleo-Pacific oceanic plate beneath the Eurasia plate, there is another collision of Indochina block with a continental fragment on the Paleo-Pacific oceanic plate - the Luconia Dangerous Ground ${ }^{11}$ by Cretaceous Figure 12.

\section{MATERIALS-METHODS}

Geochemical data analysis from rock samples collected in the specific region (A, B, C, D) Figure 1 by other researchers in Vietnam then re-used in the scope of this study to verify the relation between tectonic evolutions and their granitic magmatism. GCD (Geochemical Data Toolkit) a R language program for handling and recalculation of geochemical data, the main tool is used for the geotectonic purpose of this paper.

\section{RESULTS}

\section{Caledonian orogeny and granitoid emplace- ment}

Rock samples collected by Hieu P. T. and Hao V..in their researches ${ }^{12,13}$ zone A, Figure 1. Geochemical data analysis were performed by University of Science and Technology of China, Hefei and Geological Processes and Mineral Resources, China University of Geoscience Table 1 . 


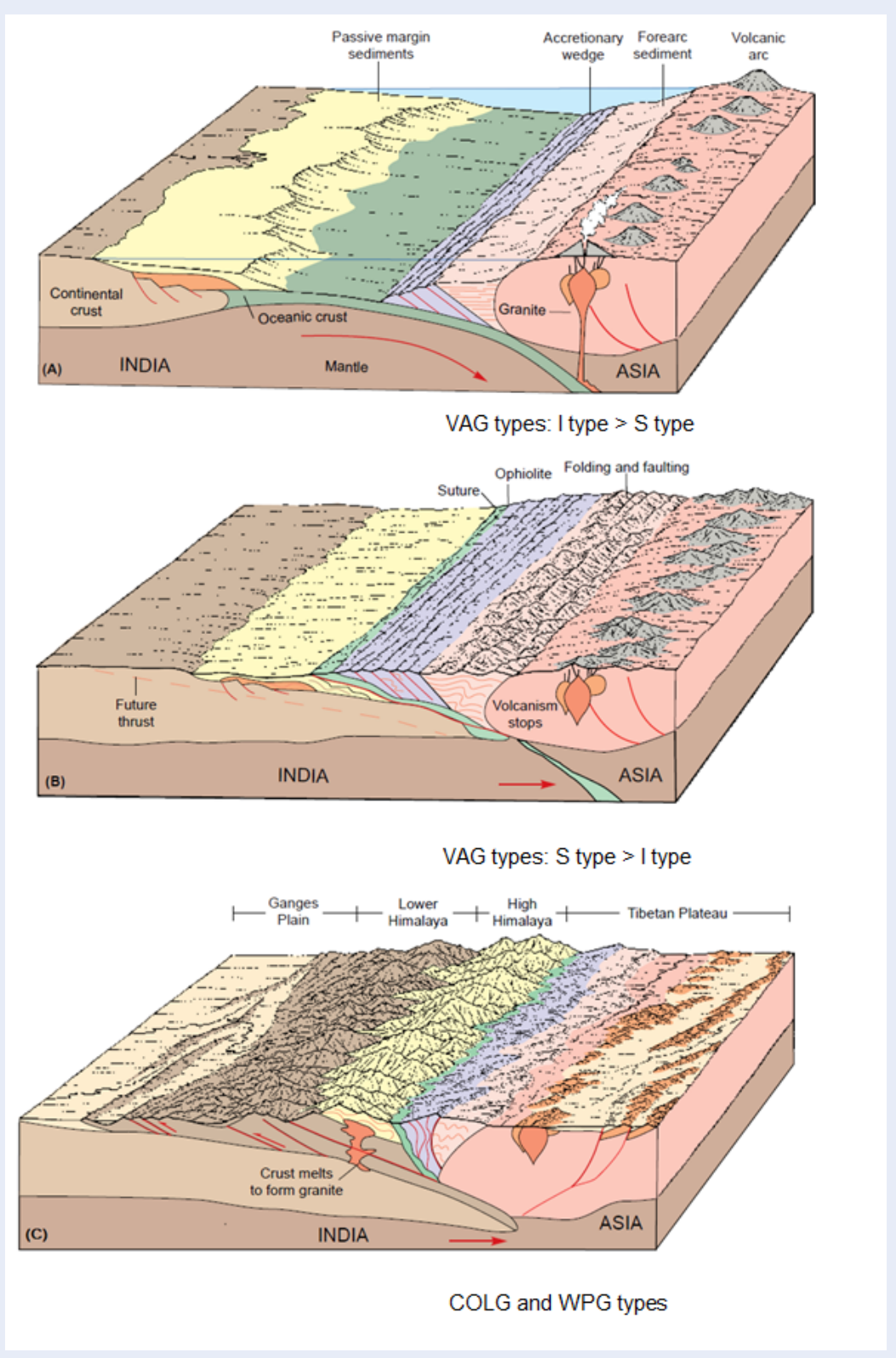

Figure 3: Evolution of a convergent tectonic and granitoid emplacement model of a collision between India and Asia. Earth Dynamic System. Chistiansen, 2002. P. 610-620. 


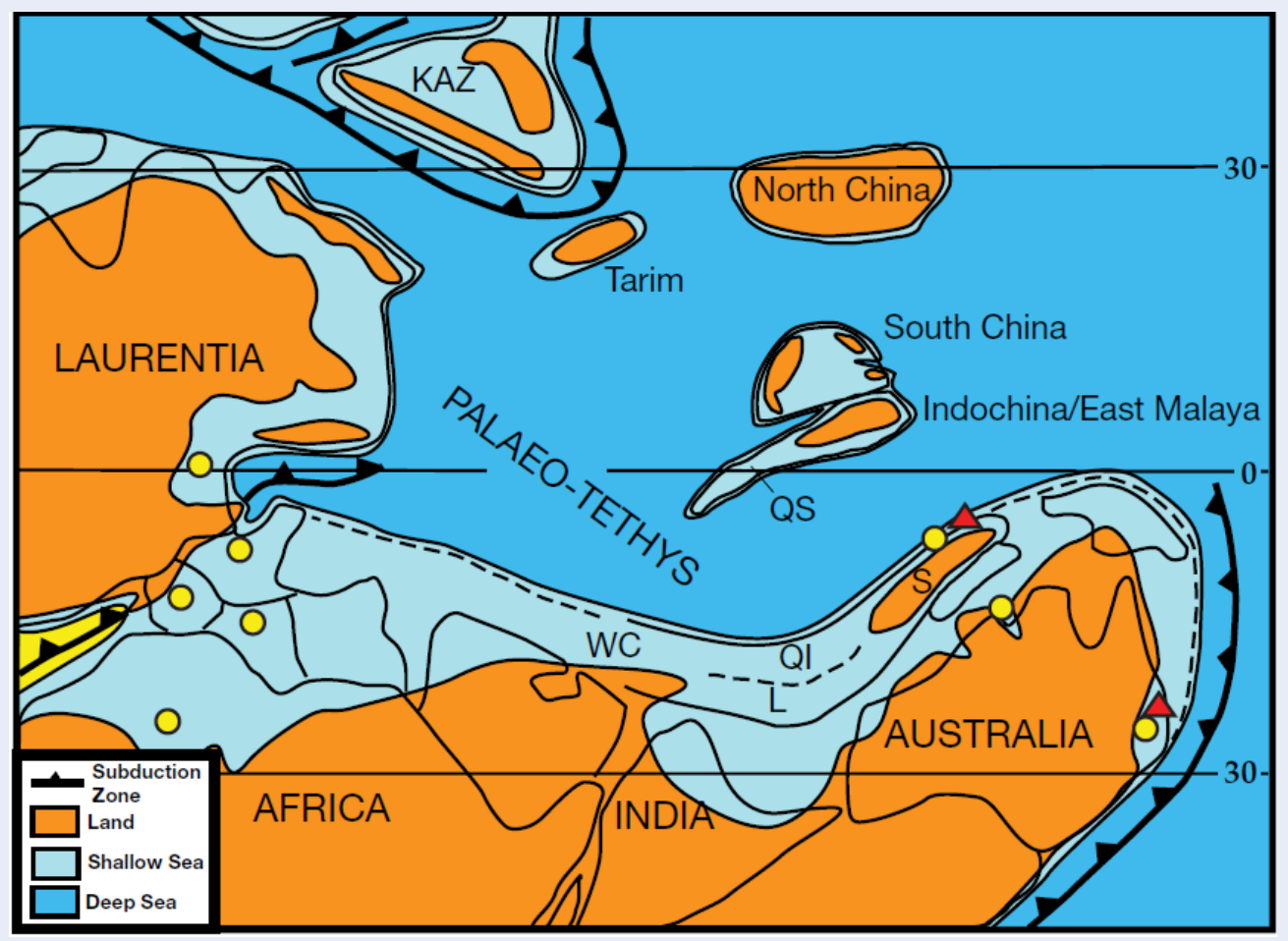

Figure 4: Cathaysialand formed by accretion of Indochina and South China by Carboniferous. Ian Metcafe, 2011.

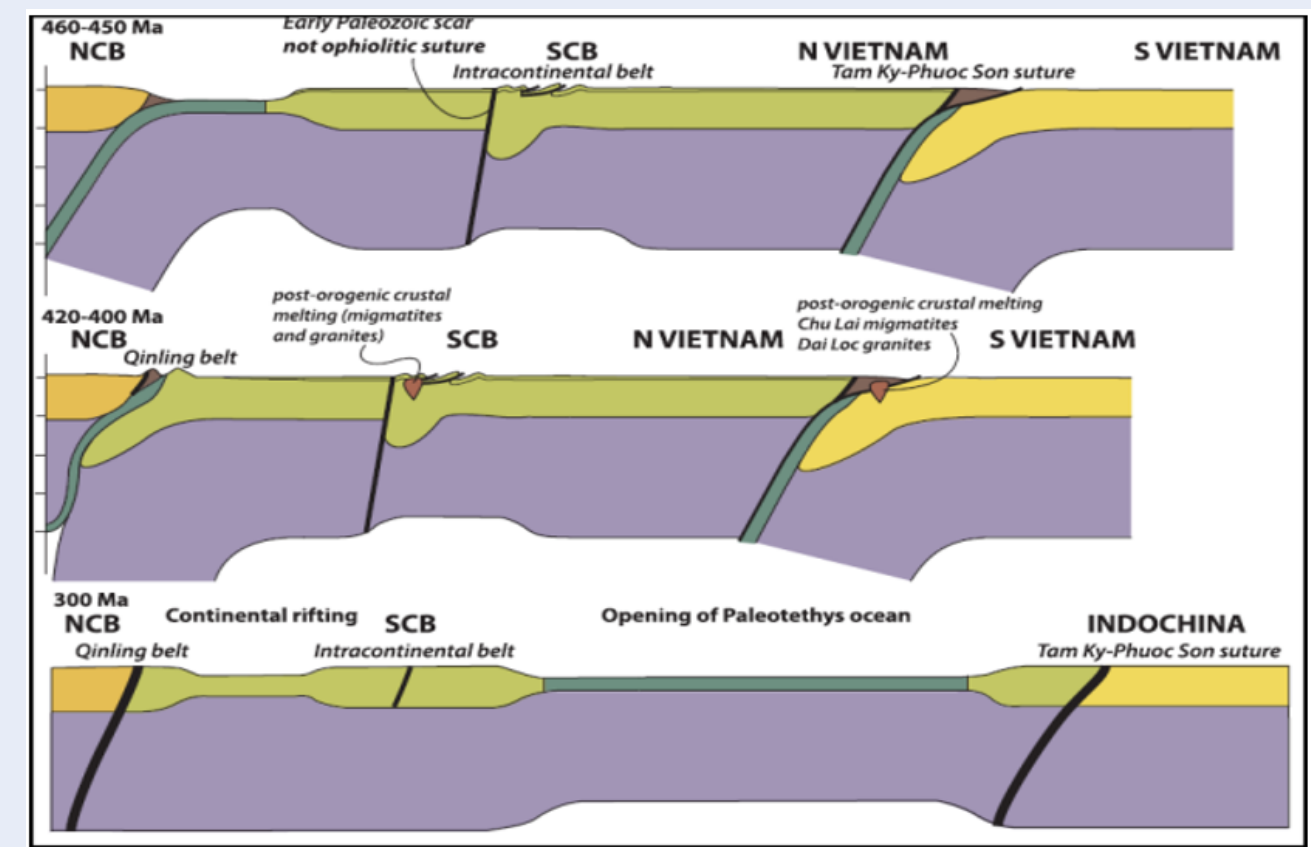

Figure 5: Tam Ky/Phuoc Son suture between N Vietnam and S Vietnam blocks. (Faure, 2018). 


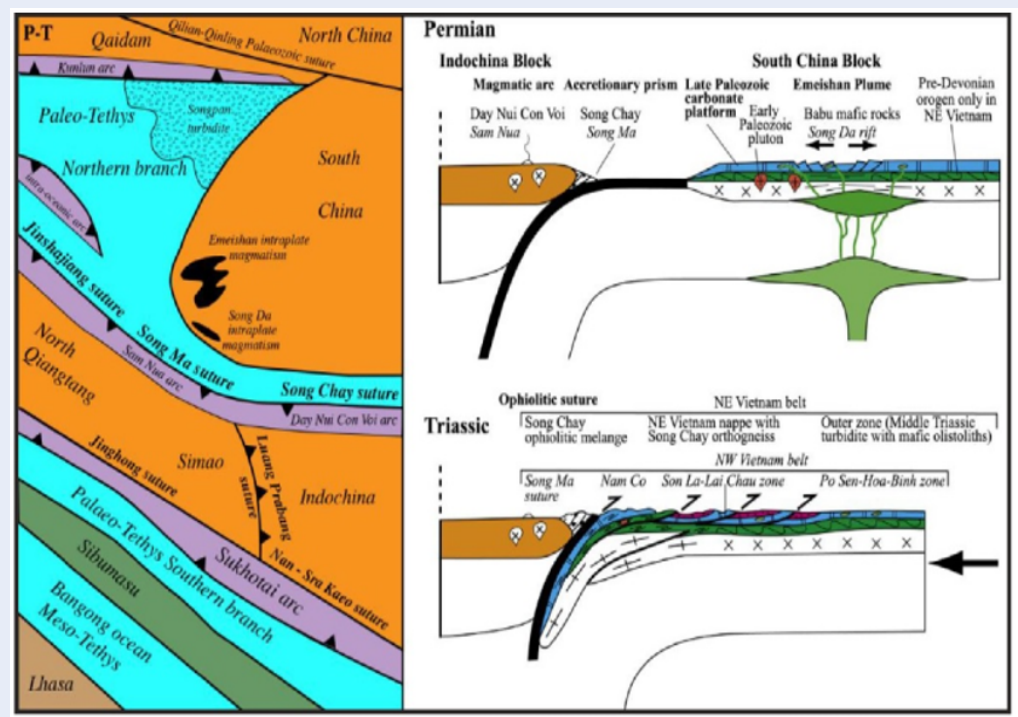

Figure 6: South China block subducted beneath Indochina block (Metcalfe, 2017).

C. Lepvrier et al./C. R. Geoscience 340 (2008) 94-111

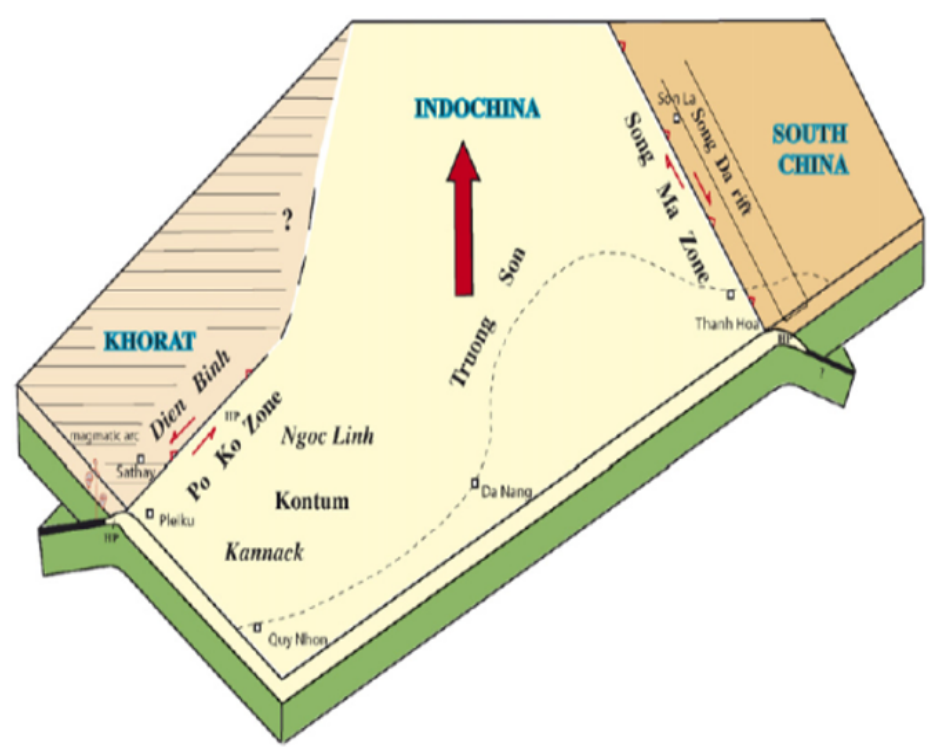

Figure 7: Indochina block subducted beneath South China block (Leprivier, 2008). 

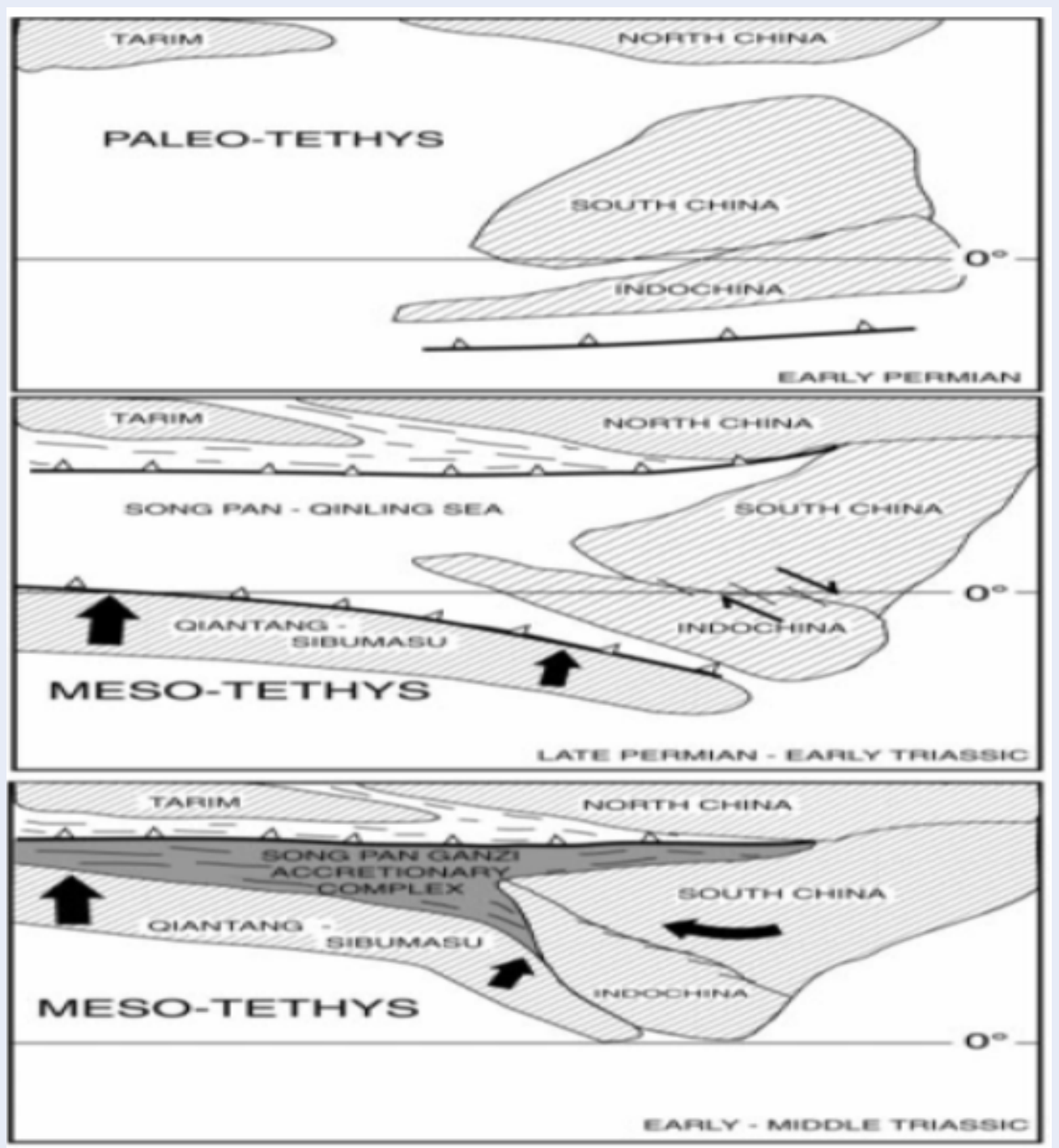

Figure 8: Reactivation of an old suture between Indochina and South China blocks (Carter, 1986). 


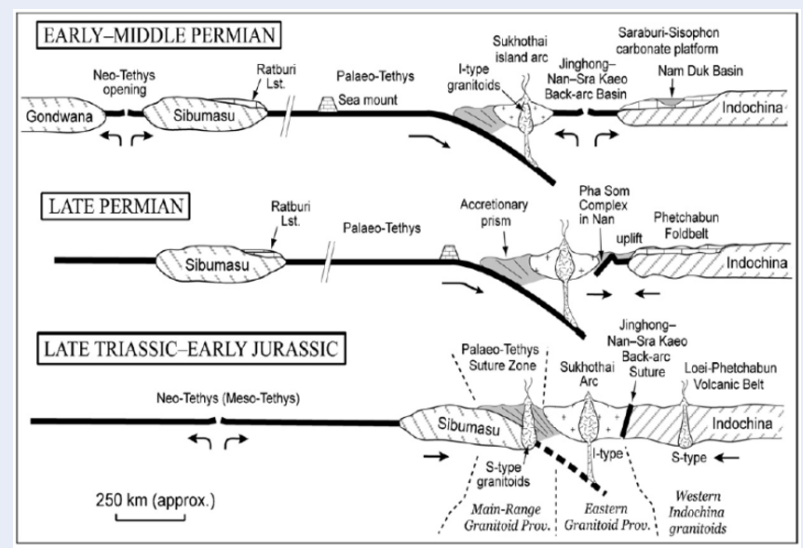

Figure 9: Sibumasu subducted beneath Indochina by early Permian (Sones and Mcalfe, 2008).

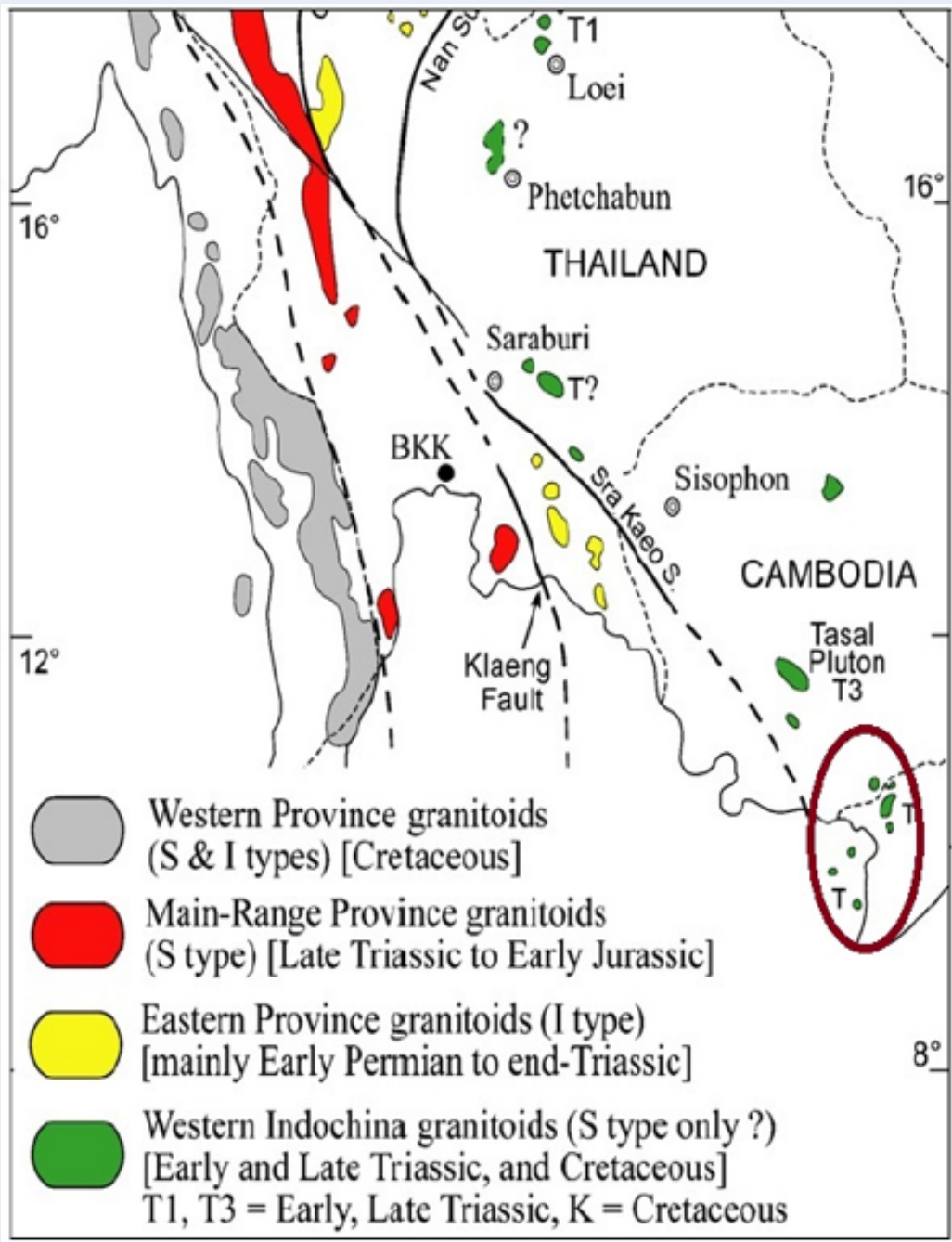

Figure 10: Granitoids belts distributed along west side of Indochina (Sones and Mcalfe, 2008). 


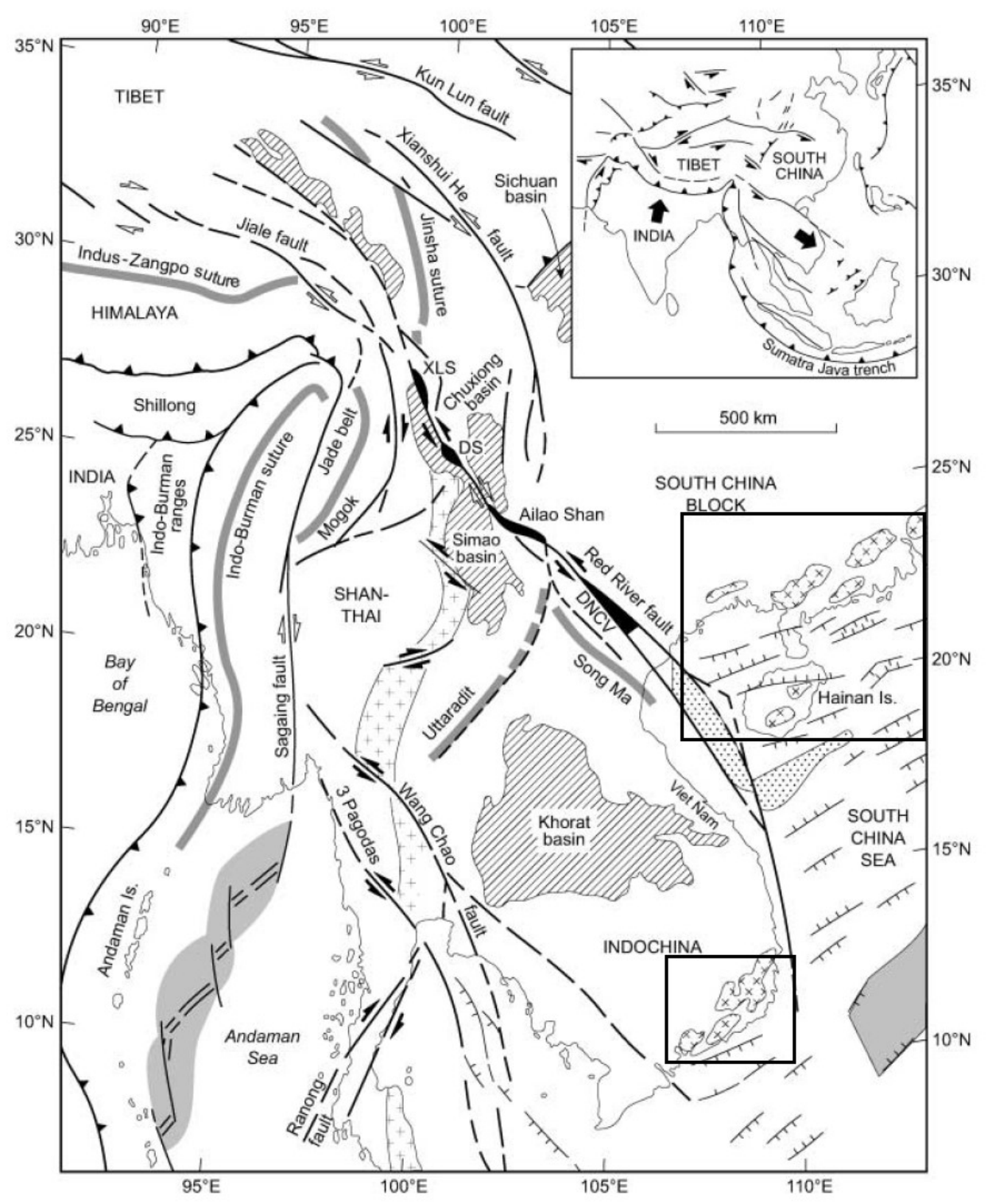

Figure 11: Yanshanian orogeny and widespread granitoid emplacements along east side of Asia (Tappoinnier, 1990, Le loupe, 1995). 


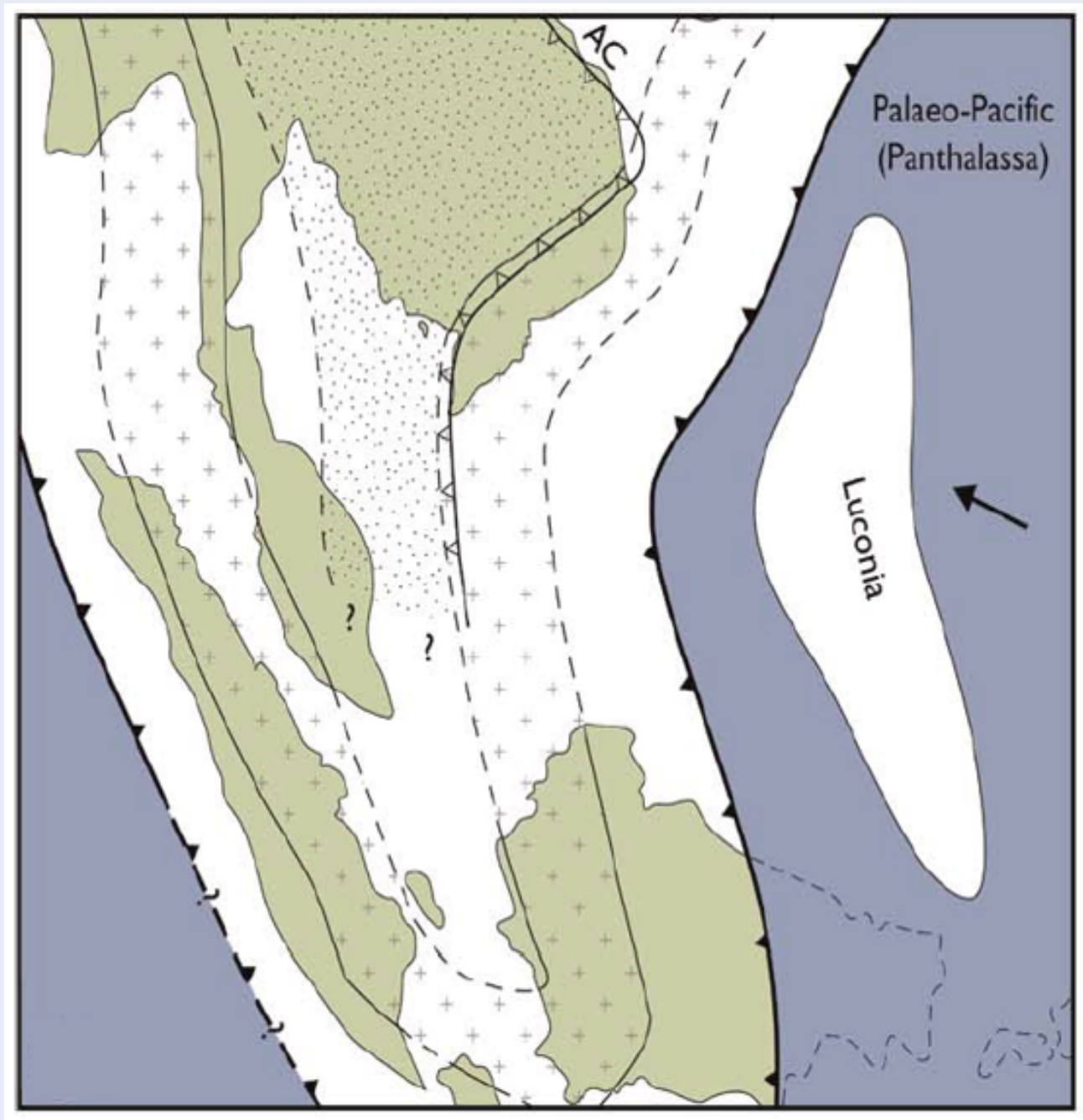

Figure 12: Luconia continental fragment on the collision with Indosinia follow Fyhn (2010). 
Table 1: Rock samples of Dai Loc and Chu Lai collected by Hieu Pham and Hao Vu (2016).

\begin{tabular}{|c|c|c|c|c|c|c|c|c|c|c|}
\hline Sample & Dai Loc & Dai Loc & Dai Loc & Dai Loc & Dai Loc & Chu Lai & Chu Lai & Chu Lai & Chu Lai & Chu Lai \\
\hline $\mathrm{SiO}_{2}$ & 70.98 & 72.38 & 71.16 & 71.21 & 70.65 & 74.78 & 74.5 & 74.46 & 73.89 & 74.28 \\
\hline $\mathrm{TiO}_{2}$ & 0.38 & 0.3 & 0.54 & 0.33 & 0.4 & 0.16 & 0.15 & 0.18 & 0.26 & 0.23 \\
\hline $\mathrm{Al}_{2} \mathrm{O}_{3}$ & 14.56 & 14.87 & 13.9 & 13.85 & 13.83 & 13.49 & 13.74 & 13.57 & 13.87 & 14.01 \\
\hline $\mathrm{Fe}_{2} \mathrm{O}_{3}$ & 3.21 & 3.02 & 3.15 & 3.17 & 3.08 & 1.34 & 1.27 & 1.42 & 1.52 & 1.26 \\
\hline $\mathrm{MnO}$ & 0.06 & 0.04 & 0.09 & 0.07 & 0.05 & 0.03 & 0.04 & 0.03 & 0.05 & 0.04 \\
\hline $\mathrm{MgO}$ & 1.12 & 0.58 & 0.67 & 0.63 & 0.51 & 0.34 & 0.25 & 0.42 & 0.36 & 0.38 \\
\hline $\mathrm{CaO}$ & 1.32 & 1.76 & 3.26 & 1.29 & 1.46 & 0.93 & 0.91 & 0.86 & 0.96 & 0.89 \\
\hline $\mathrm{Na}_{2} \mathrm{O}$ & 3.28 & 3.9 & 4.04 & 4.15 & 2.77 & 2.51 & 2.7 & 2.78 & 2.86 & 2.76 \\
\hline $\mathrm{K}_{2} \mathrm{O}$ & 4.05 & 4.98 & 5.19 & 3.97 & 5.4 & 6.13 & 5.59 & 5.56 & 5.42 & 6.21 \\
\hline $\mathrm{P}_{2} \mathrm{O}_{5}$ & 0.11 & 0.13 & 0.12 & 0.08 & 0.16 & 0.08 & 0.08 & 0.07 & 0.06 & 0.05 \\
\hline LOI & 0.89 & 0.75 & 0.68 & 0.76 & 0.66 & 0.38 & 0.52 & 0.46 & 0.65 & 0.78 \\
\hline Total & 99.97 & 102.66 & 102.84 & 99.53 & 98.94 & 100.17 & 99.75 & 99.81 & 99.9 & 100.89 \\
\hline $\mathrm{K}_{2} \mathrm{O} / \mathrm{Na}_{2} \mathrm{O}$ & 1.23 & 1.28 & 1.28 & 0.96 & 1.95 & 2.44 & 2.07 & 2 & 1.9 & 2.25 \\
\hline NCNK & 1.19 & 0.99 & 0.76 & 1.03 & 1.06 & 1.08 & 1.13 & 1.12 & 1.12 & 1.09 \\
\hline NNK & 1.49 & 1.26 & 1.13 & 1.24 & 1.33 & 1.25 & 1.31 & 1.28 & 1.31 & 1.24 \\
\hline $\mathrm{Sc}$ & 6.97 & 5.9 & 5.3 & 7.41 & 5.2 & 2.58 & 2.73 & 2.62 & 2.76 & 2.54 \\
\hline $\mathrm{V}$ & 6.87 & 6.12 & 6.44 & 6.38 & 6.65 & 6.09 & 4.84 & 5.89 & 6.17 & 5.23 \\
\hline $\mathrm{Cr}$ & 5.56 & 6.12 & 5.98 & 5.25 & 6.24 & 5.53 & 4.28 & 5.42 & 4.76 & 5.12 \\
\hline Co & 0.56 & 0.48 & 0.59 & 0.32 & 0.64 & 0.3 & 0.93 & 0.87 & 0.54 & 0.73 \\
\hline $\mathrm{Ni}$ & 2.99 & 3.02 & 3.1 & 2.95 & 3.05 & 1.57 & 3.03 & 2.12 & 2.67 & 3.01 \\
\hline $\mathrm{Cu}$ & 34 & 36 & 35.8 & 35.4 & 35 & 16.5 & 20.3 & 18.7 & 19.2 & 17.6 \\
\hline $\mathrm{Zn}$ & 48.2 & 49.6 & 50.2 & 45.8 & 52.8 & 20.1 & 24.4 & 21.2 & 22.7 & 24.2 \\
\hline $\mathrm{Ga}$ & 22.3 & 16.7 & 18.9 & 21.8 & 15.8 & 17.7 & 15.8 & 16.5 & 15.5 & 17.6 \\
\hline $\mathrm{Rb}$ & 323 & 298 & 159 & 335 & 136 & 274 & 265 & 268 & 272 & 262 \\
\hline $\mathrm{Sr}$ & 98.4 & 118.3 & 119.7 & 91.2 & 135 & 43.3 & 56 & 46.5 & 52.6 & 55.8 \\
\hline $\mathrm{Zr}$ & 312 & 298 & 256 & 318 & 242 & 119 & 128 & 124 & 129 & 116 \\
\hline $\mathrm{Nb}$ & 18 & 19.2 & 14.2 & 20.8 & 12.3 & 20.6 & 14.5 & 16.6 & 17.8 & 19.3 \\
\hline Cs & 19.3 & 18.2 & 7.9 & 19.6 & 3.5 & 7.96 & 9.85 & 8.17 & 8.32 & 9.67 \\
\hline
\end{tabular}




\begin{tabular}{|c|c|c|c|c|c|c|c|c|c|c|}
\hline \multicolumn{11}{|c|}{ Table 1 continued } \\
\hline $\mathrm{Ba}$ & 667 & 658 & 667 & 689 & 646 & 216 & 252 & 246 & 232 & 256 \\
\hline $\mathrm{Hf}$ & 7.65 & 7.98 & 6.29 & 8.55 & 5.98 & 3.68 & 3.98 & 3.58 & 3.72 & 3.64 \\
\hline $\mathrm{Ta}$ & 1.56 & 1.98 & 1.54 & 1.89 & 0.91 & 1.58 & 1.35 & 1.62 & 1.46 & 1.55 \\
\hline $\mathrm{Pb}$ & 29 & 32.9 & 27.8 & 34.3 & 21.9 & 47.1 & 50.2 & 48.2 & 49.7 & 46.9 \\
\hline Th & 22.1 & 25.08 & 25.6 & 25.3 & 18.9 & 36.4 & 35.2 & 35.8 & 37.1 & 36.2 \\
\hline $\mathrm{U}$ & 4.78 & 4.09 & 4.12 & 4.58 & 4.06 & 12.1 & 10.3 & 11.1 & 12.8 & 10.7 \\
\hline $\mathrm{La}$ & 55 & 60 & 49 & 68.2 & 44 & 44.7 & 43.8 & 44.9 & 42.3 & 46.5 \\
\hline $\mathrm{Ce}$ & 112 & 128 & 98.3 & 133 & 91.3 & 100.7 & 98.8 & 97.6 & 95.9 & 101.8 \\
\hline $\operatorname{Pr}$ & 9.98 & 17.2 & 15.6 & 14.3 & 9.63 & 10.6 & 10.4 & 9.7 & 11.2 & 10.7 \\
\hline $\mathrm{Nd}$ & 48.2 & 39.5 & 48.2 & 50.5 & 36.2 & 38 & 37.4 & 39.3 & 36.8 & 37.6 \\
\hline $\mathrm{Sm}$ & 9.78 & 8.38 & 7.98 & 9.22 & 7.03 & 8.58 & 8.51 & 9.15 & 8.26 & 8.67 \\
\hline En & 1.21 & 1.45 & 1.67 & 1.12 & 1.41 & 0.56 & 0.67 & 0.72 & 0.53 & 0.62 \\
\hline $\mathrm{Gd}$ & 7.4 & 6.87 & 7.15 & 7.93 & 6.39 & 7.8 & 8.17 & 6.9 & 7.31 & 8.26 \\
\hline $\mathrm{Tb}$ & 1.16 & 0.98 & 1.06 & 1.13 & 0.92 & 1.25 & 1.41 & 1.27 & 1.65 & 1.38 \\
\hline Dy & 5.78 & 5.98 & 6.23 & 6.32 & 5.32 & 7.19 & 8.87 & 8.12 & 7.56 & 7.84 \\
\hline Ho & 1.15 & 1.08 & 1.87 & 1.22 & 1.11 & 1.4 & 1.93 & 1.6 & 1.86 & 1.78 \\
\hline Er & 3.16 & 3.45 & 3.29 & 3.35 & 3.11 & 3.74 & 5.58 & 4.87 & 4.76 & 5.21 \\
\hline $\operatorname{Tm}$ & 0.49 & 0.47 & 0.38 & 0.5 & 0.48 & 0.57 & 0.89 & 0.76 & 0.82 & 0.65 \\
\hline $\mathrm{Yb}$ & 3.29 & 3.32 & 3.4 & 3.3 & 3.35 & 3.89 & 6.22 & 4.32 & 5.23 & 6.11 \\
\hline $\mathrm{Lu}$ & 0.48 & 0.51 & 0.47 & 0.49 & 0.52 & 0.56 & 0.89 & 0.62 & 0.67 & 0.78 \\
\hline $\mathrm{Y}$ & 36.6 & 34.9 & 35 & 35.2 & 34.2 & 43.5 & 63 & 56.8 & 62 & 58.6 \\
\hline
\end{tabular}


Geotectonic diagram by Canabis et al., 1989 on Y, $\mathrm{La}$, and $\mathrm{Nb}$ and Harris et al., 1986 on Rb, Hf and Ta showed as Figure 13.

Most of the samples fall into active margin between two blocks; some can be seen go further into the collision between two blocks.

Around Devonian time, the S Vietnam block believed to subduct beneath the $\mathrm{N}$ Vietnam, which at that time belongs to the South China Block ${ }^{14}$. The tectonic activity continued to bring those two plates close to each other, and finally, weld together formed the Cathaysialand at the end Carbonifereous ${ }^{15}$. The almagamation of these two blocks not so intense some syn-collision granites could be found, but post COL granites could not be found. The boundary between these two blocks is suggested as Tam Ky - Phuoc Son suture Figure 1.

\section{Indosinian orogeny and granitoid emplace- ment}

Indosinian is an important tectonic event in Indochina block; within Vietnam, many studies are conducted by Vietnamese and foreign geologists. Result of this tectonic Indochina block welded to South China block, and the suture is confirmed by the Song Ma ophiolitic belt of Triassic time. Rock samples were collected by Pham T. H., zone B, Figure 1, and results of geochemical analysis were performed by the Institute of Geology and Geophysics, Chinese Academy and re-used in this study Table $2^{16}$.

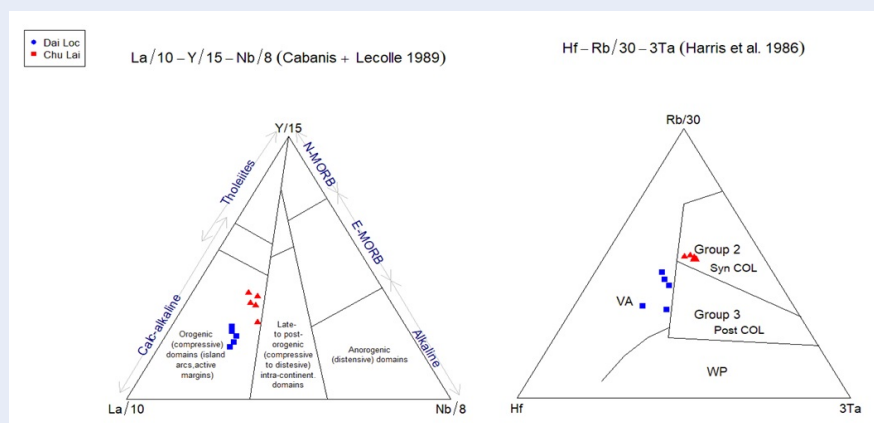

Figure 13: Plots of Dai Loc, and Chu Lai granites VA (Volcanic Arc granite), COL (Collision granite), WP (Within Plate granite). 
Table 2: Rock samples collected in Ben Giang and Que Son by Hieu Pham (2015).

\begin{tabular}{|c|c|c|c|c|c|c|}
\hline Samples & Hai Van & Hai Van & Hai Van & Hai Van & Ben Giang & Ben Giang \\
\hline $\mathrm{SiO}_{2}$ & 70.4 & 70.32 & 74.53 & 72.04 & 74.05 & 72.35 \\
\hline $\mathrm{TiO}_{2}$ & 0.4 & 0.52 & 0.24 & 0.46 & 0.23 & 0.25 \\
\hline $\mathrm{Al}_{2} \mathrm{O}_{3}$ & 14.73 & 14.39 & 13.62 & 13.22 & 14.2 & 14.71 \\
\hline $\mathrm{Fe}_{2} \mathrm{O}_{3} \mathrm{t}$ & 2.63 & 4.83 & 1.93 & 3.73 & 0.39 & 2.01 \\
\hline $\mathrm{MnO}$ & 0.03 & 0.08 & 0.03 & 0.05 & 0.04 & 0.05 \\
\hline $\mathrm{MgO}$ & 0.73 & 2.15 & 0.87 & 1.44 & 0.32 & 0.63 \\
\hline $\mathrm{CaO}$ & 2.35 & 0.87 & 1.06 & 1.33 & 1.02 & 1.05 \\
\hline $\mathrm{Na}_{2} \mathrm{O}$ & 2.8 & 1.2 & 1.91 & 1.71 & 3.24 & 3.25 \\
\hline $\mathrm{K}_{2} \mathrm{O}$ & 4.25 & 3.93 & 4.97 & 4.17 & 4.45 & 4.84 \\
\hline $\mathrm{P}_{2} \mathrm{O}_{5}$ & 0.09 & 0.09 & 0.11 & 0.16 & 0.16 & 0.21 \\
\hline LOI & 0.38 & 1.16 & 1.06 & 1.2 & 1.84 & 0.78 \\
\hline Total & 98.79 & 99.54 & 100.33 & 99.51 & 99.94 & 100.13 \\
\hline $\mathrm{K}_{2} \mathrm{O} / \mathrm{Na}_{2} \mathrm{O}$ & 1.52 & 3.28 & 2.6 & 2.44 & 1.37 & 1.49 \\
\hline $\mathrm{A} / \mathrm{CNK}$ & 1.09 & 1.84 & 1.3 & 1.35 & 1.18 & 1.18 \\
\hline $\mathrm{A} / \mathrm{NK}$ & 1.6 & 2.31 & 1.6 & 1.8 & 1.4 & 1.39 \\
\hline Sc & 7.93 & 10.6 & 4.63 & 9.21 & 2.09 & 3.98 \\
\hline V & 14 & 73.7 & 29.5 & 52.4 & 615 & 17.8 \\
\hline $\mathrm{Cr}$ & 11.9 & 65.3 & 25.7 & 45.9 & 624 & 15.8 \\
\hline Co & 1.51 & 9.92 & 3.89 & 7.43 & 110 & 2.67 \\
\hline $\mathrm{Ni}$ & 7.52 & 26.2 & 11.2 & 20.4 & 267 & 7.57 \\
\hline $\mathrm{Cu}$ & 18.6 & 28.5 & 18.5 & 36.7 & 29.6 & 47.3 \\
\hline $\mathrm{Zn}$ & 67.6 & 7.75 & 4.89 & 65.9 & 61.9 & 71.9 \\
\hline $\mathrm{Ga}$ & 17.4 & 20 & 15.2 & 19 & 19.1 & 21.6 \\
\hline $\mathrm{Rb}$ & 219 & 223 & 257 & 223 & 227 & 205 \\
\hline $\mathrm{Sr}$ & 154 & 73.9 & 88.2 & 90.2 & 41.2 & 83.1 \\
\hline $\mathrm{Zr}$ & 158 & 170 & 119 & 229 & 112 & 125 \\
\hline $\mathrm{Nb}$ & 13.6 & 18.8 & 9.88 & 15.2 & 13.7 & 14 \\
\hline Cs & 10.4 & 13.7 & 12 & 11.6 & 30 & 22.2 \\
\hline
\end{tabular}




\begin{tabular}{|c|c|c|c|c|c|c|}
\hline \multicolumn{7}{|c|}{ Table 2 continued } \\
\hline $\mathrm{Ba}$ & 806 & 638 & 644 & 581 & 196 & 419 \\
\hline Hf & 4.56 & 4.61 & 3.45 & 6.56 & 2.06 & 3.51 \\
\hline $\mathrm{Ta}$ & 1.11 & 1.24 & 0.9 & 1.4 & 2.01 & 1.68 \\
\hline $\mathrm{Pb}$ & 22 & 13.1 & 32 & 32.6 & 46 & 41.7 \\
\hline Th & 19.6 & 26.8 & 14.6 & 24.7 & 8.71 & 19.4 \\
\hline $\mathrm{U}$ & 4.29 & 5.93 & 6.49 & 5.98 & 7.5 & 3.35 \\
\hline $\mathrm{La}$ & 38.9 & 52.7 & 27.2 & 42.7 & 13.6 & 30.3 \\
\hline $\mathrm{Ce}$ & 80.2 & 107 & 55 & 84.9 & 30.4 & 65 \\
\hline $\operatorname{Pr}$ & 8.91 & 11.7 & 5.95 & 9.23 & 3.27 & 6.84 \\
\hline $\mathrm{Nd}$ & 34.1 & 43.9 & 21.9 & 34.4 & 12.1 & 25.3 \\
\hline Sm & 7.27 & 8.38 & 4.62 & 6.95 & 3.15 & 5.26 \\
\hline $\mathrm{Eu}$ & 1.76 & 0.98 & 1.15 & 1.09 & 0.49 & 0.74 \\
\hline Gd & 7.01 & 7.13 & 4.31 & 6.14 & 2.92 & 4.44 \\
\hline $\mathrm{Tb}$ & 1.11 & 0.97 & 0.7 & 0.91 & 0.45 & 0.6 \\
\hline Dy & 6.8 & 5.39 & 4.25 & 5.26 & 2.18 & 3.11 \\
\hline Ho & 1.47 & 1.05 & 0.9 & 1.04 & 0.35 & 0.57 \\
\hline Er & 4.28 & 2.88 & 2.63 & 2.92 & 0.8 & 1.47 \\
\hline $\mathrm{Tm}$ & 0.65 & 0.41 & 0.42 & 0.45 & 0.11 & 0.22 \\
\hline $\mathrm{Yb}$ & 4.27 & 2.71 & 2.86 & 3.07 & 0.74 & 1.47 \\
\hline $\mathrm{Lu}$ & 0.64 & 0.4 & 0.43 & 0.47 & 0.11 & 0.21 \\
\hline $\mathrm{Y}$ & 45.4 & 31.4 & 28.3 & 31.3 & 11 & 17.9 \\
\hline$(\mathrm{La} / \mathrm{Yb})_{N}$ & 6.1 & 13 & 6.4 & 9.3 & 12.3 & 13.8 \\
\hline$(\mathrm{Tb} / \mathrm{Yb})_{N}$ & 1.15 & 1.58 & 1.08 & 1.31 & 2.69 & 1.8 \\
\hline $\mathrm{Eu} / \mathrm{Eu}^{*}$ & 0.76 & 0.39 & 0.79 & 0.51 & 0.5 & 0.47 \\
\hline
\end{tabular}


In geotectonic diagrams, almost all of the samples fall into the field of the active continental margin, and some samples could be found in the collision zone Figure 14.

The convergence between Indochina and South China blocks produces both VAG and COLG granitoid rocks. It may not clearly demonstrate the full scope of tectonic evolution in detail, but it may prove that the tectonic activities composed of the first stage of subduction continued with the next stage of the collision of these two blocks. It is suggested that South China was subducted beneath the Indochina block based on most of Ben Giang and Hai Van granitoids have stayed in the southern part of Song Ma suture, the location where two blocks amalgamated together Figure 1.

\section{Indochina/Sibumasu amalgamation and granitoid emplacement}

At the same time in the west side of the Indochina block, the Sibumasu block, after broken up from Gondwanaland, came and subducted beneath the Indochina block. Granitoid emplacements for this tectonic evolution were found in a long time span from the early Permian toward the end of Cretaceous time from northern Thailand toward the south to the Malaysia peninsula. In Vietnam, a conductive study by the HCM University of Science with the cooperative of Academia Sinica, Taiwan, by Ching Yin Lan (2009) to collect samples of the Nui Cam batholith in the SW corner Vietnam. Rock analysis was performed at the Academia Sinica, EPMA laboratory. Research study has been presented in the Goldschmith Geology Conferences, Canada 2009.

In the geotectonic plots, these samples are falling mostly in the field of VAG, Syn-COLG, and WPG. The Sibumasu block came and subducted beneath the Indochina block, the tectonic activities and granitoid emplacement could be found at the first stage of subduction with VAG and later on with Syn COLG and WPG in the next phase of collision COLG between two plates and finally induced a type of within plate granitoid WPG in the last phase, Figure 15.

\section{Yanshangnian orogeny and granitoid em- placement}

By the Cretaceous time, the Paleo-Pacific oceanic plate subducted beneath the Asia continental plate, this type of convergence produced a broad and long granitoid belt from north China toward the south to Vietnam as Truong Son belt. Rock samples collected in Dalat zone by Bich Thuy N. T. and analysis performed by Memorial University of St. John's Newfoundland, $\mathrm{U} \mathrm{K} .{ }^{17}$, Table 3 . However, the nature of this tectonic event is not simple with just a merely subduction of the Paleo-Pacific oceanic crust beneath the Eurasia continental crust. It is more complex as ${ }^{10}$ suggestion that there is a continental fragment that possibly on the Paleo-Pacific plate, following the course of subduction, this block came to collide with Indochina block Figure 16. 
$\mathrm{La} / 10-\mathrm{Y} / 15-\mathrm{Nb} / 8$ (Cabanis + Lecolle 1989)

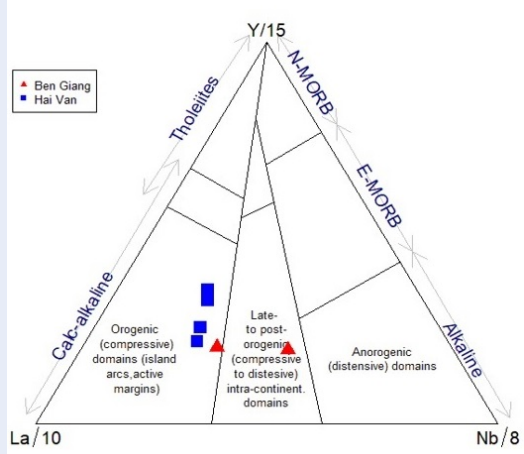

$\mathrm{Hf}-\mathrm{Rb} / 30-3 \mathrm{Ta}$ (Harris et al. 1986)

Figure 14: Geotectonic plots: Hai Van (S>I type/VGA); Ben Giang (I type/COLG)

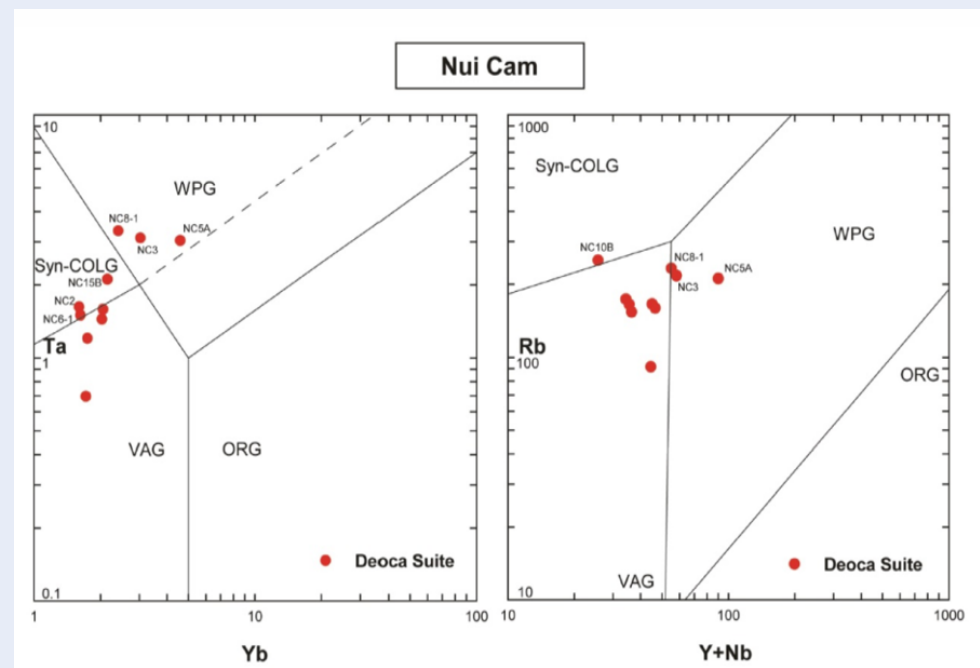

Figure 15: Geotectonic plots: Dinh Quan (I type/VAG); Ca Na, Deo Ca (I type/COLG).

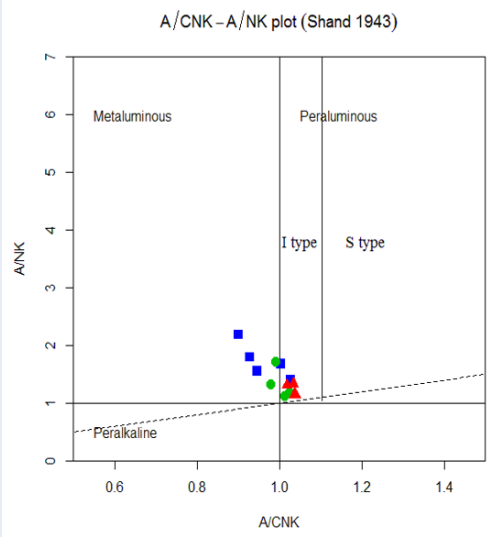

$\mathrm{Hf}-\mathrm{Rb} / 30-3 \mathrm{Ta}$ (Harris et al. 1986)

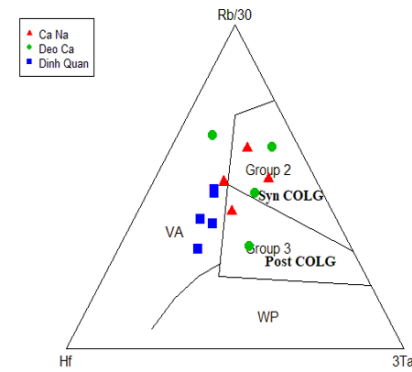

Figure 16: Dinh Quan fall mostly in the field of VAG, and $\mathrm{Ca} \mathrm{Na}$, Deo Ca belong to COLG the stage, seeing as the result of collision between the Indochina and the referred Luconia block. 

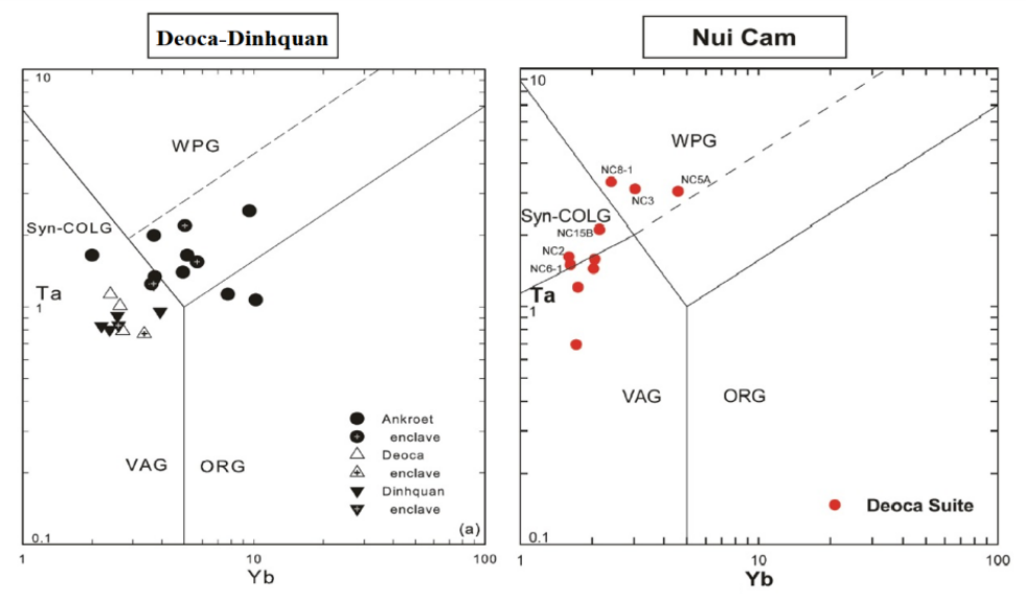

Figure 17: Differences between DeoCa-Dinh Quan and Nui Cam trace elements plot.

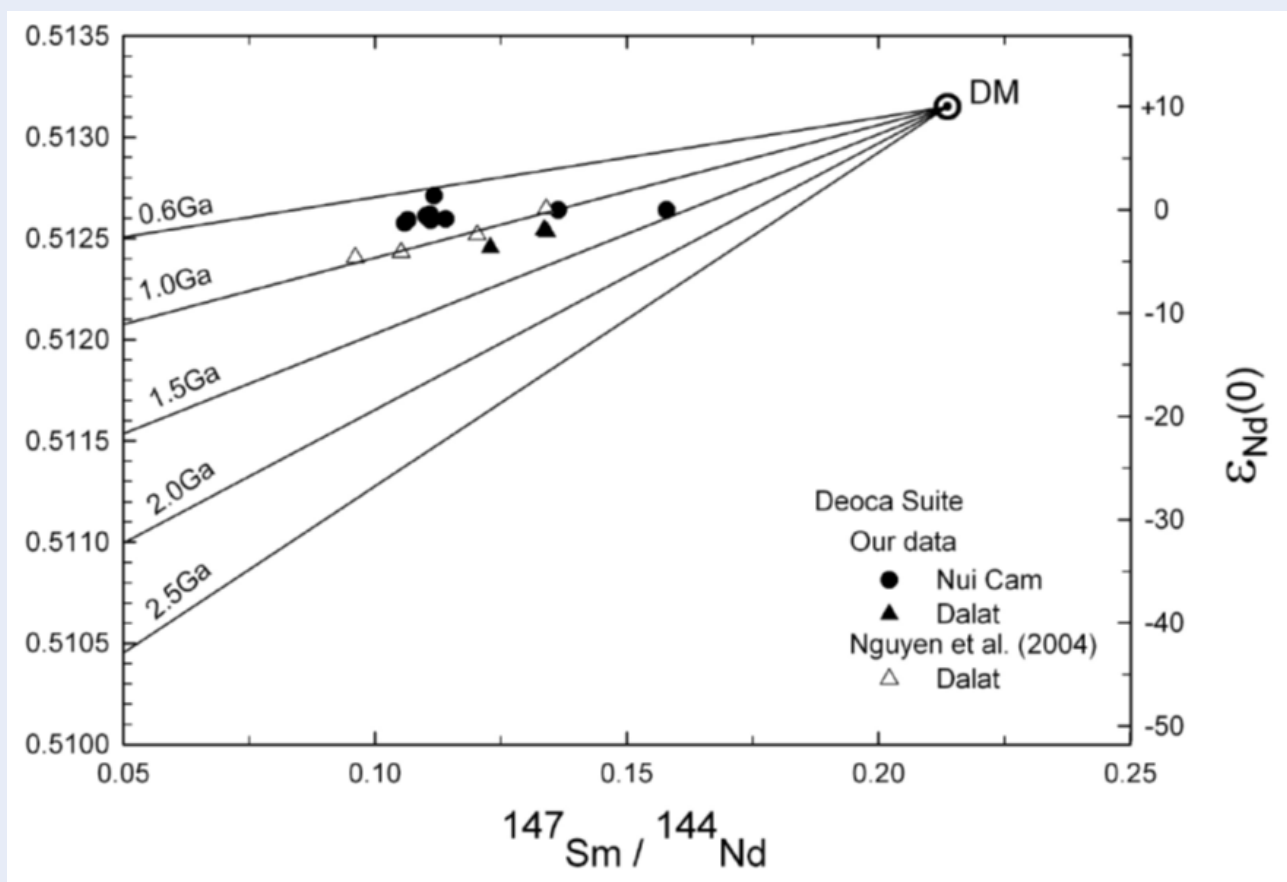

Figure 18: Differences of Tdm ages of Dalat and NuiCam granitoids, Lan (2009). 
Table 3: Rock samples collected in the Dalat zone by Bich Thuy N. T (2004).

\begin{tabular}{|c|c|c|c|c|c|c|c|c|c|c|c|c|c|}
\hline Samples & DQ 1 & DQ 2 & DQ 3 & DQ 4 & DQ 5 & $\mathrm{DC} 1$ & DC 2 & DC 3 & $\mathrm{DC} 4$ & $\mathrm{CN} 1$ & $\mathrm{CN} 2$ & $\mathrm{CN} 3$ & $\mathrm{CN} 4$ \\
\hline $\mathrm{SiO}_{2}$ & 58.01 & 63.82 & 70.01 & 67.32 & 68.2 & 68.21 & 76.32 & 70.71 & 76.62 & 77.12 & 73.25 & 77.81 & 73.51 \\
\hline $\mathrm{TiO}_{2}$ & 1.02 & 0.61 & 0.22 & 0.5 & 0.31 & 0.4 & 0.12 & 0.5 & 0.11 & 0.13 & 0.21 & 0.12 & 0.22 \\
\hline $\mathrm{Al}_{2} \mathrm{O}_{3}$ & 17.34 & 15.13 & 15.06 & 14.81 & 15.14 & 15.42 & 12.71 & 14.35 & 12.42 & 12.65 & 13.62 & 12.51 & 13.74 \\
\hline $\mathrm{Fe}_{2} \mathrm{O}_{3}$ & 7.02 & 5.81 & 3.04 & 3.92 & 3.71 & 3.6 & 1.14 & 2.72 & 1.61 & 1.41 & 1.9 & 1.02 & 2.05 \\
\hline $\mathrm{MnO}$ & 0.12 & 0.12 & 0.11 & 0.07 & 0.08 & 0.05 & 0.01 & 0.06 & 0.05 & 0.02 & 0.02 & 0.01 & 0.03 \\
\hline $\mathrm{MgO}$ & 2.81 & 2.4 & 0.52 & 1.61 & 1.2 & 1.41 & 0.11 & 0.77 & 0.05 & 0.02 & 0.25 & 0.05 & 0.28 \\
\hline $\mathrm{CaO}$ & 6.23 & 4.33 & 2.11 & 3.35 & 3.32 & 3.62 & 0.95 & 2.11 & 0.62 & 0.61 & 1.62 & 0.61 & 1.62 \\
\hline $\mathrm{Na}_{2} \mathrm{O}$ & 3.11 & 2.81 & 4.05 & 2.95 & 3.25 & 3.06 & 3.32 & 3.61 & 3.8 & 3.52 & 3.24 & 3.15 & 3.71 \\
\hline $\mathrm{K}_{2} \mathrm{O}$ & 2.61 & 3.52 & 3.84 & 4.34 & 3.42 & 3.64 & 4.83 & 4.52 & 4.51 & 4.9 & 4.71 & 5.31 & 3.91 \\
\hline $\mathrm{P}_{2} \mathrm{O}_{5}$ & 0.32 & 0.21 & 0.11 & 0.11 & 0.21 & 0.11 & 0.13 & 0.15 & 0.03 & 0.01 & 0.06 & 0.01 & 0.06 \\
\hline $\mathrm{H}_{2} \mathrm{O}$ & 0.52 & 0.7 & 0.41 & 0.52 & 0.5 & 1.13 & 0.21 & 0.3 & 0.3 & 0.31 & 0.51 & 0.42 & 0.61 \\
\hline Total & 99.11 & 99.46 & 99.68 & 99.18 & 99.34 & 100.6 & 99.85 & 99.92 & 100.1 & 100.7 & 99.89 & 100 & 99.73 \\
\hline ASI & 0.9 & 0.92 & 1.02 & 0.96 & 1.01 & 0.99 & 1.03 & 0.97 & 1.02 & 1.04 & 1.03 & 1.05 & 1.04 \\
\hline Co & 0 & 0.02 & 0.5 & 0.01 & 0.42 & 0.11 & 0.41 & 0.11 & 0.32 & 0.61 & 0.5 & 0.6 & 0.55 \\
\hline $\mathrm{Cr}$ & 87 & 71 & 91 & 157 & 112 & 5 & 21 & 40 & 134 & 28 & 19 & 30 & 23 \\
\hline $\mathrm{Ni}$ & 51 & 31 & 32 & 28 & 35 & 2 & 25 & 19 & 27 & 24 & 20 & 16 & 22 \\
\hline $\mathrm{Sr}$ & 474 & 285 & 246 & 233 & 404 & 306 & 79 & 302 & 38 & 8 & 127 & 22 & 235 \\
\hline $\mathrm{Ba}$ & 465 & 393 & 553 & 295 & 543 & 401 & 118 & 566 & 218 & 10 & 262 & 16 & 443 \\
\hline $\mathrm{Zr}$ & 183 & 160 & 209 & 160 & 124 & 129 & 99 & 224 & 112 & 101 & 133 & 99 & 106 \\
\hline $\mathrm{Rb}$ & 97 & 153 & 146 & 210 & 109 & 113 & 304 & 211 & 180 & 291 & 264 & 351 & 150 \\
\hline $\mathrm{Hf}$ & 4.76 & 3.42 & 4.9 & 4.51 & 3.51 & 3.54 & 3.61 & 0.89 & 2.52 & 2.41 & 4.71 & 3.11 & 3.51 \\
\hline $\mathrm{Nb}$ & 8 & 5.3 & 5.6 & 7.1 & 6.2 & 11.1 & 12.2 & 10.5 & 11.5 & 11.1 & 6.2 & 4.1 & 7.5 \\
\hline $\mathrm{Ta}$ & 0.83 & 0.71 & 0.8 & 0.92 & 0.76 & 1.51 & 0.53 & 1.12 & 1.32 & 1.19 & 1.2 & 2.52 & 1.1 \\
\hline Th & 10.3 & 12.6 & 15.6 & 29.2 & 10.6 & 14.4 & 41.3 & 30.5 & 16.4 & 25.7 & 29.6 & 46.4 & 14.3 \\
\hline $\mathrm{U}$ & 2.25 & 1.87 & 2.36 & 5.41 & 3.12 & 3.91 & 20.32 & 4.45 & 3.69 & 7.66 & 8.82 & 14.7 & 8.83 \\
\hline $\mathrm{Y}$ & 25 & 27 & 28 & 26 & 16 & 20 & 21 & 26 & 29 & 51 & 48 & 76 & 24 \\
\hline $\mathrm{Zn}$ & 71 & 65 & 50 & 44 & 64 & 56 & 15 & 44 & 37 & 40 & 46 & 30 & 31 \\
\hline $\mathrm{La}$ & 24.3 & 25.9 & 32.3 & 28.2 & 22.4 & 21.8 & 21.1 & 42.7 & 19.3 & 19.7 & 29.1 & 38.6 & 22.6 \\
\hline
\end{tabular}




\begin{tabular}{|c|c|c|c|c|c|c|c|c|c|c|c|c|c|}
\hline \multicolumn{14}{|c|}{ Table 3 continued } \\
\hline $\mathrm{Ce}$ & 50.7 & 53.1 & 60.5 & 55.4 & 41.4 & 42.6 & 38.9 & 82.4 & 41.5 & 45.6 & 59.8 & 77.5 & 44 \\
\hline $\operatorname{Pr}$ & 6.01 & 6.16 & 6.84 & 6.13 & 4.5 & 4.71 & 3.76 & 8.8 & 4.8 & 5.81 & 6.98 & 10.9 & 4.91 \\
\hline $\mathrm{Nd}$ & 23.6 & 23.3 & 24.8 & 22.1 & 16.3 & 17 & 11.6 & 30.8 & 17.7 & 24 & 26.5 & 40 & 17.7 \\
\hline $\mathrm{Sm}$ & 5.04 & 4.92 & 4.91 & 4.62 & 3.21 & 3.42 & 1.9 & 5.32 & 3.89 & 6.5 & 6.14 & 8.87 & 3.81 \\
\hline $\mathrm{Eu}$ & 1.5 & 0.91 & 0.9 & 0.81 & 0.8 & 0.8 & 0.22 & 1.01 & 0.32 & 0.11 & 0.5 & 0.31 & 0.5 \\
\hline $\mathrm{Gd}$ & 4.42 & 4.33 & 4.08 & 3.86 & 2.53 & 2.96 & 1.51 & 4.2 & 3.61 & 6.32 & 5.82 & 6.59 & 3.31 \\
\hline $\mathrm{Tb}$ & 0.65 & 0.62 & 0.63 & 0.63 & 0.42 & 0.51 & 0.23 & 0.51 & 0.52 & 1.04 & 1.01 & 0.87 & 0.52 \\
\hline Dy & 3.91 & 4.1 & 3.82 & 3.45 & 2.24 & 2.84 & 1.6 & 3.22 & 3.3 & 6.68 & 6.31 & 4.76 & 3.21 \\
\hline Ho & 0.83 & 0.95 & 0.85 & 0.7 & 0.51 & 0.62 & 0.43 & 0.61 & 0.71 & 1.4 & 1.32 & 0.9 & 0.75 \\
\hline Er & 2.52 & 2.76 & 2.61 & 2.35 & 1.4 & 1.9 & 1.41 & 1.8 & 2.02 & 4.41 & 4.25 & 2.5 & 2.1 \\
\hline $\mathrm{Tm}$ & 0.36 & 0.43 & 0.44 & 0.32 & 0.21 & 0.32 & 0.22 & 0.35 & 0.31 & 0.64 & 0.61 & 0.41 & 0.31 \\
\hline $\mathrm{Yb}$ & 2.12 & 2.45 & 2.41 & 2.01 & 1.35 & 1.81 & 1.75 & 1.71 & 2.19 & 3.9 & 3.72 & 2.51 & 1.95 \\
\hline $\mathrm{Lu}$ & 0.34 & 0.41 & 0.4 & 0.32 & 0.23 & 0.33 & 0.31 & 0.31 & 0.41 & 0.59 & 0.5 & 0.42 & 0.32 \\
\hline
\end{tabular}




\section{DISCUSSION}

Geochemical data of magma samples collected in the central and Granitoid emplacements in Thailand also in the Malaysia peninsula have been viewed as products of tectonic evolution of the region Figure 5, and they have been seen by different granitoid belts e.g., Western, Main range, Eastern, and Western Indochina Figure 6 . Not many studies on Nui Cam batholiths, located at the corner of southwest Vietnam, zone D Figure 1; however, Nui Cam granitoic rocks were defined and classified as Deo Ca and Dinh Quan complex based merely on geographical studies also on geochronological studies. Rock with the similarities mineral assemblage is classified as the same group (Granitoids emplacement map in southern Viet Nam, 2004. HCM University of Science).

Recent studies in the region, these granitoids may have belonged to the Western Indochina Belt Figures 5 and 6 granitic rocks with ages vary from early Permian to Early Cretaceous. It is clearly different between two groups, on the geotectonic plot Deoca and Dinhquan complex belong to VAG while Nui Cam moves to the field of Syn-COLG or WPG Figure 17. More than that Tdm determination of these two groups ${ }^{16,18}$ showing they are different between Dalat (1.2 Ga) compare to Nuicam (0.8 Ga) Figure 18.

\section{CONCLUSION}

Tectonic evolutions in Vietnam are very complex and complicated. It is not evolved to the intereaction between two single geological blocks but more than two. Many studies and research have been done in the region to bring out the whole picture of tectonic evolution of Vietnam It's likely suitable for major tectonic activities with theirs granitoid emplacement through space and time. Granitoids ages and their geotectonic features seem to be agreeable with the relative location compare with the boundary between them. However more works need to be conducted to clarify Nuicam batholith, it may not belong to Deoca/Dinhquan suite as previously classified, but it may belong to another system, the Western Indochina granitoid belt.

\section{COMPETING INTERESTS}

The author declares that this paper has no competing interests.

\section{ACKNOWLEDGMENT}

The authors would like to thank for the authorization of Dr. Nguyen B Thuy and Dr. Pham T Hiếu for using their geochemical data and their great supports for this paper.

\section{REFERENCES}

1. Winter J. Principles of Igneous and Metamorphic Petrology. 2010;p. 194-197.

2. Tuấn NA, Sang HN. Địa Kiến Tạo Đại Cương, nhà xuất bản Đại Học Quốc Gia TPHCM. 2018;p. 44-49.

3. Tong-Dzuy T, et al. Fish suggests continental connections between Indochina and South China blocks in Middle Devonian time. Geology. 1996;

4. Metcalfe I. Mesozoic-Paleozoic history of SE Asia. 2011;Available from: https://doi.org/10.1144/SP355.2.

5. Krobicki, et al. Major Tectonic Events and Plates of Northwest Vietnam. 2006:

6. Lepvrier, et al. Indosinian Tectonics in Vietnam. Geoscience 2008 . 2007;

7. Hutchison. Geological Evolution of South-east Asia. 1989;

8. Carter, et al. Understanding Mesozoic Accretion in Southeast Asia: significant of Triassic thermotectonism in Vietnam. Geology. 2001;Available from: https://doi.org/10.1130/00917613(2001)029<0211:UMAISA>2.0.CO;2.

9. Sones and Metcalfe. Paralell Tethyan sutures in mainland Southeast Asia: New insights for Paleo-Tethys closure and implications for the Indosinian orogeny. 2007; Available from: https://doi.org/10.1016/j.crte.2007.09.008.

10. Searly MP. Role of the Red River Shear Zone, Yan Nam, and Vietnam in the Continental Extrusion of Southeast Asia. Journal of Geology Society London. 2006;

11. Fyhn, et al. Palaeocence-Early Eocence inversion of the Phuquoc-Kampot Som basin: SE Asian deformation associated with the suturing of Luconia. Journal of Geological Society, London, 2010. 2010;Available from: https://doi.org/10. 1144/0016-76492009-039.

12. Pham $\mathrm{TH}$, et al. U-Pb ages and $\mathrm{Hf}$ isotopic composition of zircon and bulk rock geochemistry of the Dai Loc granitoid complex in Kontum massif: Implications for early Paleozoic crustal evolution in Central Vietnam. Journal of Mineralogical and Petrological Sciences. 2016;

13. Hao V. Thesis Assertion, HCMUT, 2016. 2016;.

14. Faure. The South China Block-Indochina collision: where, when, and how? . Journal of Southeast Asia Earth Science. 2014;Available from: https://doi.org/10.1016/j.jseaes.2013.09. 022.

15. Faure. Triassic tectonics of the southern margin of the South China Block. C R Geoscience. 2016;348. Available from: https: //doi.org/10.1016/j.crte.2015.06.012.

16. Pham TH. Late Permian to Early Triassic crustal evolution of the Kontum massif, central Vietnam: Zircon U-Pb ages and geochemical and $\mathrm{Nd}-\mathrm{Hf}$ isotopic composition of the Hai Van granitoid complex International Geology Review. 2015;.

17. Lan CY, et al. Characteristics of late Mesozoic rocks in SW corner of Southern Vietnam. Goldsmith, Canada. 2010;

18. Nguyen, et al. Geochemical and isotopic constraints on the petrogenesis of granitoids from the Dalat zone, southern Vietnam. Journal of Asian Earth Sciences. 2004;23. Available from: https://doi.org/10.1016/j.jseaes.2003.06.001. 